MATHEMATICS OF COMPUTATION

Volume 78, Number 265, January 2009, Pages 387-403

S $0025-5718(08) 02131-5$

Article electronically published on May 16, 2008

\title{
UNIMODULAR LATTICES IN DIMENSIONS 14 AND 15 OVER THE EISENSTEIN INTEGERS
}

\author{
KANAT ABDUKHALIKOV AND RUDOLF SCHARLAU
}

\begin{abstract}
All indecomposable unimodular hermitian lattices in dimensions 14 and 15 over the ring of integers in $\mathbb{Q}(\sqrt{-3})$ are determined. Precisely one lattice in dimension 14 and two lattices in dimension 15 have minimal norm 3.
\end{abstract}

In 1978 W. Feit 10 classified the unimodular hermitian lattices of dimensions up to 12 over the ring $\mathbb{Z}[\omega]$ of Eisenstein integers, where $\omega$ is a primitive third root of unity. These lattices all have roots, that is, vectors of norm 2. In dimension 13, for the first time a unimodular lattice without roots appears [1, 3. In 2] the unimodular lattices in dimension 13 are completely classified. The root-free lattice turns out to be unique. It has minimal norm 3, and its automorphism group is isomorphic to the group $\mathbb{Z}_{6} \times \operatorname{PSp}_{6}(3)$ of order $2^{10} .3^{10} .5 .7 .13$. The remaining lattices all have roots; the rank of the root system is 12 in all cases.

In this paper, we classify the unimodular lattices in dimensions 14 and 15 . There are exactly 58 , respectively 259 classes of indecomposable lattices in these dimensions. Below, we list their root systems and the orders of their automorphism groups. Gram matrices for all lattices are available electronically via

www .mathematik. uni-dortmund.de/ scharlau

There is only one root-free unimodular lattice of rank 14 , and there are two root-free unimodular lattices of rank 15.

The lattices without roots have minimal norm 3; they are extremal as introduced for unimodular Eisenstein lattices in 8, Chapter 10.7. They give rise to 3-modular extremal $\mathbb{Z}$-lattices in twice the dimension, as defined by Quebbemann in 17 . See 8, 19, 20 for more information on extremal and modular lattices and their relation to modular forms. In this context, the lattices classified in this paper can be considered as complex structures on (extremal) 3-modular lattices. The question for existence, uniqueness, and possibly a full classification of extremal modular lattices has been an ongoing challenge, both computationally and theoretically, after the appearance of the influential paper [17].

Let $V$ be a vector space over $\mathbb{Q}(\sqrt{-3})$ with a positive definite hermitian product (, ). A lattice $L$ in $V$ is a finitely generated $\mathbb{Z}[\omega]$-module contained in $V$ such that $L$ contains a basis of $V$ and $(x, y) \in \mathbb{Z}[\omega]$ for all $x, y \in L$. More precisely, one

Received by the editor October 19, 2007 and, in revised form, January 2, 2008.

2000 Mathematics Subject Classification. Primary 11H06, 11H56; Secondary 11E39, 11H71, $11 \mathrm{~F} 11$.

Key words and phrases. Integral lattice, hermitian lattice, extremal lattice, unimodular lattice, root system.

The first author was supported by the Alexander von Humboldt Foundation. 
calls this an integral lattice. The ring $\mathbb{Z}[\omega]$ is a principal ideal domain. Thus every finitely generated torsion-free $\mathbb{Z}[\omega]$-module is free. The discriminant $d(L)$ of $L$ is the determinant of the Gram matrix $\left(a_{i}, a_{j}\right)$ with respect to some basis $a_{1}, \ldots, a_{n}$ of $L$. The lattice is unimodular if $d(L)=1$. The norm of a vector $x \in L$ is $N(x)=(x, x)$. The minimal norm of a lattice $L$ is $\min \{N(x, x) \mid x \in L, x \neq 0\}$. Let $L^{(2)}$ denote the sublattice of $L$ generated by all vectors of norm 2 in $L$, the so-called roots of $L$, and let $\mu_{2}(L)$ denote the number of roots in $L$. Finally, $G(L)$ denotes the group of all automorphisms of $L$ which preserve the form. The group $G(L)$ is finite. Every lattice can be uniquely decomposed into an orthogonal sum of orthogonally indecomposable lattices (it can be proved by standard reasoning similar to one in [12]). In view of the known classification in smaller dimensions it is therefore sufficient to list the indecomposable lattices.

We now come to the actual construction of unimodular lattices. We use various methods, such as representations of finite groups, lifting of linear codes, neighbour steps at suitable primes, and computer-assisted as well as hand computations of various kinds. We use extensively Schiemann's computer program 21] for calculation of invariants of hermitian lattices and their neighbours, and the Magma Computational Algebra System [4. The crucial point is the computation of automorphism groups, which (in both systems) is based on the work [16. After we have constructed (and distinguished) sufficiently many lattices, we use the mass formula as worked out in 10 to check the completeness of our list. An easy to calculate but important invariant of our lattices is their root systems. Due to the well-known classification of complex reflection groups [22, 5], there exist a few additional root systems over $\mathbb{Z}[\omega]$ which are not defined over $\mathbb{Z}$. Of course, they are familiar from the work on lattices of smaller dimension cited above. We use the following standard notation for root lattices. $I_{n}$ denotes a lattice of rank $n$ with an orthonormal basis. Equivalently,

$$
I_{n}=\left\langle\left(a_{1}, \ldots, a_{n}\right) \mid a_{i} \in \mathbb{Z}[\omega]\right\rangle
$$

and if $x=\left(a_{1}, \ldots, a_{n}\right), y=\left(b_{1}, \ldots, b_{n}\right)$, then $(x, y)=\sum_{i=1}^{n} a_{i} \bar{b}_{i}$. The lattices $A_{n} \subseteq I_{n+1}, E_{8}, E_{7}$ and $E_{6}$ are as usual. For $\alpha \in \mathbb{Z}[\omega]$ define $D_{n}(\alpha) \subseteq I_{n}$ by

$$
D_{n}(\alpha)=\left\langle\left(a_{1}, \ldots, a_{n}\right) \mid a_{i} \in \mathbb{Z}[\omega], \sum_{i=1}^{n} a_{i} \equiv 0 \quad(\bmod \alpha)\right\rangle .
$$

Finally,

$$
\begin{gathered}
U_{5}=\left\langle A_{5},(1 / \sqrt{-3})\left(1, \omega, \omega^{2}, 1, \omega, \omega^{2}\right)\right\rangle, \\
U_{6}=\left\langle D_{6}(\sqrt{-3}),(1 / \sqrt{-3})(1, \ldots, 1)\right\rangle .
\end{gathered}
$$

The lattice $U_{6}$ is unimodular, and $U_{5}$ has discriminant 2 .

The root systems of the lattices classified in this paper are given in Tables 1-3 below. For most of the lattices in dimension 14, we give "glue vectors" (additional generators) 8 together with the root systems.

In Table 1 we have collected separately all lattices that are constructed from self-dual codes of length 14 over $\mathbb{F}_{4}$. These codes have been classified in [6, 14. Let $\varphi$ denote the composite mapping $\mathbb{Z}[\omega] \rightarrow \mathbb{Z}[\omega] / 2 \mathbb{Z}[\omega] \cong \mathbb{F}_{4}$. Then for any self-dual code $C$ of length 14 over $\mathbb{F}_{4}$ the lattice

$$
L=\frac{1}{\sqrt{2}}\left\langle\left(a_{1}, \ldots, a_{14}\right) \in I_{14} \mid\left(\varphi\left(a_{1}\right), \ldots, \varphi\left(a_{14}\right)\right) \in C\right\rangle
$$


is a unimodular lattice of rank 14. In particular, lattice No. 10 comes from a quadratic residue code [13, 23] of length 14 .

Now we come to a particular construction of a unimodular lattice of rank 15.

Lemma 1. The exterior square $U_{6} \wedge U_{6}$ is a unimodular lattice of rank 15 and minimal norm 3.

Proof. We only need to show that the lattice $U_{6} \wedge U_{6}$ has minimal norm 3. Our proof is similar to the proof of Theorem 2.1 in [9]. Any element $a \in U_{6} \wedge U_{6}$ can be written in the form $a=\sum_{i=1}^{r} x_{i} \wedge y_{i}$, where $r \leq 3$ and $x_{1}, \ldots, x_{r}, y_{1}, \ldots, y_{r}$ are $2 r$ linearly independent vectors in $U_{6}$. The lattice $L_{2 r}$ generated by $x_{1}, \ldots, x_{r}$, $y_{1}, \ldots, y_{r}$ depends only on $a$. We have

$$
\begin{aligned}
(a, a) & =\left(\sum_{i=1}^{r} x_{i} \wedge y_{i}, \sum_{j=1}^{r} x_{j} \wedge y_{j}\right) \\
& =\sum_{i, j}\left[\left(x_{i}, x_{j}\right)\left(y_{i}, y_{j}\right)-\left(x_{i}, y_{j}\right)\left(y_{i}, x_{j}\right)\right] \\
& =\operatorname{Tr}(A \bar{B}-C \bar{C}),
\end{aligned}
$$

where $A=\left(\left(x_{i}, x_{j}\right)\right)_{1 \leq i, j \leq r}, B=\left(\left(y_{i}, y_{j}\right)\right)_{1 \leq i, j \leq r}$ and $C=\left(\left(x_{i}, y_{j}\right)\right)_{1 \leq i, j \leq r}$. Define

$$
H=\left(\begin{array}{cc}
A & C \\
{ }^{t} \bar{C} & B
\end{array}\right), J=\left(\begin{array}{cc}
0 & I_{r} \\
-I_{r} & 0
\end{array}\right) .
$$

Then $H$ is the hermitian matrix of the lattice $L_{2 r}$ and

$$
(a, a)=\frac{1}{2} \operatorname{Tr}\left(H^{t} \overline{J H} J\right) \geq\left.\left.\frac{2 r}{2}|H|^{1 / 2 r} \cdot\right|^{t} \overline{J H} J\right|^{1 / 2 r}=r|H|^{1 / r},
$$

where we used the inequality $\operatorname{Tr}(M N) \geq m|M|^{1 / m}|N|^{1 / m}$ valid for any hermitian positive definite matrices $M, N$ of degree $m$ (it can be proved similarly to the proof of Lemma 7.1.3 in [11]). Now we show that $(a, a) \geq 3$. Indeed, for $r=3$ we have $(a, a) \geq 3 .|H|^{1 / 3} \geq 3$; for $r=2$ we have $(a, a) \geq 2 .|H|^{1 / 2}>2$; for $r=1$ we have $(a, a) \geq 1 .|H|=d\left(L_{2 r}\right) \geq 3$. Finally, it is easy to construct an element $a$ of norm 3: take $a=x \wedge y$ with $(x, x)=(y, y)=2$ and $(x, y)=1$.

Theorem 1. There are 58 hermitian indecomposable unimodular lattices over $\mathbb{Z}[\omega]$ of dimension 14. They are listed in Tables 1 and 2. One of them has no roots; the remaining lattices have root systems of ranks $6,8,10,11,12,13$ and 14 . The root-free lattice $L_{0}$ has minimal norm 3 and automorphism group $\mathbb{Z}_{6} \times \mathrm{G}_{2}(3) .2$ of order $2^{8} \cdot 3^{7} .7 .13$.

Theorem 2. There are 259 hermitian indecomposable unimodular lattices over $\mathbb{Z}[\omega]$ of dimension 15. They are listed in Table 3. For any integer $r$ from 0 to 15 there is an indecomposable unimodular lattice of dimension 15 with a root system of rank $r$. There are only two root-free lattices. They have minimal norm 3. One of the rootfree lattices is isometric to the exterior square $U_{6} \wedge U_{6}$ and has automorphism group $\mathbb{Z}_{2} \times 3 . \mathrm{U}_{4}(3) .2$ of order $2^{9} .3^{7} .5 .7$. The second root-free lattice has automorphism group $\mathbb{Z}_{2} \times\left(3_{+}^{1+2} \times 3_{+}^{1+2}\right) . \mathrm{SL}_{2}(3) .2$ of order $2^{5} .3^{7}$.

Proof. Let $\mathfrak{G}_{n}$ be a complete set of representatives of the isomorphism classes of lattices of rank $n$ and discriminant 1 . Let $\mathfrak{G}_{n}^{0}$ be the set of all those lattices in $\mathfrak{G}_{n}$ 
that contain no elements of norm 1. Define

$$
\begin{aligned}
M_{n} & =\sum_{L \in \mathfrak{G}_{n}} \frac{1}{|G(L)|}, \quad M_{n}(2)=\sum_{L \in \mathfrak{G}_{n}} \frac{\mu_{2}(L)}{|G(L)|}, \\
Y_{n} & =\sum_{L \in \mathfrak{G}_{n}^{0}} \frac{1}{|G(L)|}, \quad Y_{n}(2)=\sum_{L \in \mathfrak{G}_{n}^{0}} \frac{\mu_{2}(L)}{|G(L)|} .
\end{aligned}
$$

These quantities can be calculated in principle well-known formulas: the mass formula for positive definite hermitian lattices, and a similar formula for average representation numbers like $\mu_{2}$. In our situation these formulas have been made explicit in [10]:

$$
\begin{gathered}
\frac{M_{2 m}}{M_{2 m-1}}=(-1)^{m-1} B_{2 m} \frac{3^{m}+(-1)^{m}}{4 m}, \quad \frac{M_{2 m+1}}{M_{2 m}}=\frac{a_{2 m+1}}{3^{m}+(-1)^{m}}, \\
\frac{M_{2 m}(2)}{M_{2 m}}=\frac{4 m\left(2^{2 m-1}+1\right)}{\left(3^{m}+(-1)^{m}\right) B_{2 m}}, \quad \frac{M_{2 m+1}(2)}{M_{2 m+1}}=\frac{\left(2^{2 m}-1\right)\left(3^{m}-(-1)^{m}\right)}{a_{2 m+1}}, \\
Y_{n}=M_{n}-\frac{1}{n ! 6^{n}}-\sum_{j=1}^{n-1} \frac{Y_{j}}{(n-j) ! 6^{n-j}}, \\
Y_{n}(2)=M_{n}(2)-\sum_{j=1}^{n-1} \frac{Y_{j}(2)}{(n-j) ! 6^{n-j}}-\sum_{j=1}^{n-2} \frac{Y_{j}}{2(n-2-j) ! 6^{n-2-j}}-\frac{1}{2(n-2) ! 6^{n-2}},
\end{gathered}
$$

where $a_{2 m+1}=3^{2 m}(-1)^{m-1} B_{2 m+1}\left(\frac{1}{3}\right) /(2 m+1), B_{n}(t)$ is the Bernoulli polynomial, and $B_{2 m}(0)=B_{2 m}$ is the Bernoulli number $B_{2 m}$. By definition $M_{1}=1 / 6$ and $M_{1}(2)=Y_{1}=Y_{1}(2)=0$.

Then one can calculate

$$
\begin{aligned}
& M_{14}=\frac{689532652191539}{2^{25} \cdot 3^{19} \cdot 5^{3} \cdot 11}, \quad M_{14}(2)=\frac{1722885336811913}{2^{23} \cdot 3^{17} \cdot 5^{3} \cdot 11}, \\
& Y_{14}=\frac{902121810728981}{2^{24} \cdot 3^{14} \cdot 5^{3} \cdot 7^{2} \cdot 11 \cdot 13}, \quad Y_{14}(2)=\frac{321547203435163}{2^{22} \cdot 3^{12} \cdot 5^{3} \cdot 11 \cdot 13} \text {. } \\
& M_{15}=\frac{4366489808207046403}{2^{26} .3^{21} \cdot 5^{3} \cdot 11}, \quad M_{15}(2)=\frac{1884491476714586441}{2^{24} .3^{18} \cdot 5^{3} .11}, \\
& Y_{15}=\frac{3619970721202760389}{2^{18} \cdot 3^{20} \cdot 5^{3} \cdot 7^{2} \cdot 11 \cdot 13}, \quad Y_{15}(2)=\frac{312324214206248801}{2^{16} \cdot 3^{17} \cdot 5^{2} \cdot 7^{2} \cdot 11 \cdot 13} .
\end{aligned}
$$

Now we use an explicit list of Gram matrices of the constructed lattices. We use Schiemann's computer program and the Magma Computational Algebra System to calculate $\mu_{2}(L)$ and $|G(L)|$ in each case. Then a straightforward check of the mass formulas for $Y_{14}$ and $Y_{15}$ confirms the completeness of our list. The formulas for $Y_{14}(2)$ and $Y_{15}(2)$ give an additional check (ignoring the lattices without roots).

We now determine the structure of the automorphism groups of the three rootfree lattices. The group $G_{2}(3) .2$ has a representation of dimension 14 over $\mathbb{Q}(\sqrt{-3})$ (see [7]) and it acts on a lattice $L$ of rank 14 over $\mathbb{Z}[\omega]$. Now results of [15] on rational maximal finite matrix groups in dimension 28 imply that this lattice is unimodular, and therefore is isometric to our root-free lattice $L_{0}$. The factor $\mathbb{Z}_{6}$ in $G\left(L_{0}\right)$ comes from roots of unity in $\mathbb{Z}[\omega]$.

Now we come to the automorphism group of the root-free lattice $U_{6} \wedge U_{6}$ introduced in Lemma 1. Recall that $\operatorname{Aut}\left(U_{6}\right) \cong 6 . \mathrm{U}_{4}(3) .2$. There is a natural homomorphism $\varphi: \operatorname{Aut}\left(U_{6}\right) \mapsto \operatorname{Aut}\left(U_{6} \wedge U_{6}\right)$, given by $\varphi(\sigma)(x \wedge y)=\sigma(x) \wedge \sigma(y)$ 
with Ker $\varphi=\{ \pm$ id $\}$. Therefore, $\operatorname{Aut}\left(U_{6} \wedge U_{6}\right) \supseteq 3 . \mathrm{U}_{4}(3) .2$. But $\{ \pm \mathrm{id}\} . \varphi\left(\operatorname{Aut}\left(U_{6}\right)\right)$ is a group of order $2^{9} \cdot 3^{7} \cdot 5.7$. The known orders of the two automorphism groups of root-free lattices in dimension 15 show that this group must be the full group. Therefore, $\operatorname{Aut}\left(U_{6} \wedge U_{6}\right) \cong \mathbb{Z}_{2} \times 3 . \mathrm{U}_{4}(3) .2$.

The structure of the automorphism group of the second root-free lattice, of order $2^{5} .3^{7}$ was determined with the help of Magma.

The extremal 3 -modular $\mathbb{Z}$-lattice in dimension 28 coming from our root-free lattice in dimension 14 appeared for the first time in [15] since its automorphism group is a rational irreducible maximal finite subgroup of $\mathrm{GL}_{28}(\mathbb{Q})$. The two rootfree lattices of rank 15 have been found by A. Schiemann ([21], also mentioned in [19]), using a sophisticated computer search for lattices with a large minimum.

We want to add some comments on algebraic techniques for lattices which are related in one way or another to the automorphism group. One of the most important algebraic techniques for lattices is "gluing", which in the most general case means: (re)constructing a lattice $L$ from a known, "simpler" sublattice $M$. Then $L$ is generated by $M$ and certain additional vectors, called glue vectors. If $M$ has the same rank as $L$, that is, generates the vector space $V$, the glue vectors must come from the dual lattice $M^{\#}$, which can be analyzed once for all. Typically, $M$ is decomposable while $L$ is not, so the additional vectors "glue the components of $M$ together", this is where the terminology comes from. An important choice for $M$ is the lattice $L^{(2)}$ generated by the roots. Then $M$ is characteristic, that is, $\operatorname{Aut}(L)$ acts on $M$, and is a subgroup of $\operatorname{Aut}(M)$ if $M$ is of full rank. If $M$ is not of full rank, it must be glued with a lattice on its orthogonal subspace. Here are two cases where the rank of the root system is very small.

Let us consider the unimodular 15-dimensional lattice $\Lambda$ (No. 257) with root system $A_{2}$. This lattice is obtained by gluing of a 2-dimensional lattice with root system $A_{2}$ and a 13-dimensional lattice $\Lambda^{\prime}$ with discriminant 3. Computations on Magma and [7] show that $\operatorname{Aut}(\Lambda) \cong 6 .\left(\mathrm{S}_{3} \times \mathrm{PSL}_{2}(27) .3\right)$. Therefore, $\operatorname{Aut}\left(\Lambda^{\prime}\right) \cong$ 6.(PSL $\left.\mathrm{PS}_{2}(27) .3\right)$ and $\Lambda^{\prime}$ is associated with the irreducible complex character of the group $\mathrm{PSL}_{2}(27)$ of degree 13 . It is the Weil character and it can indeed be realized over $\mathbb{Z}[\omega]$ (see [18]).

Similar reasoning shows that the 15-dimensional unimodular lattice No. 259 with root system $A_{1}$ has automorphism group $6 .\left(\mathrm{S}_{2} \times \mathrm{PSL}_{2}(13)\right)$ and it is obtained from a 14-dimensional lattice of discriminant 2 with automorphism group $6 . \mathrm{PSL}_{2}(13)$. This lattice generates an irreducible complex character of the group $\mathrm{PSL}_{2}(13)$ of degree 14 (the case of complex character of degree 13 is excluded since the description 1] of hermitian lattices of dimension 13 associated with the irreducible complex character of the group $\mathrm{PSL}_{2}(13)$ of degree 13 shows that this case is impossible).

Of course, root sublattices and their complements are not the only choice for characteristic sublattices. If the automorphism group is already known, one could proceed as in the following example. The complex representation associated with the action of the automorphism group $\mathbb{Z}_{2} \times\left(3_{+}^{1+2} \times 3_{+}^{1+2}\right) \cdot \mathrm{SL}_{2}(3) .2$ on the 15 dimensional root-free lattice $L_{2}$ is reducible, and is a sum of two irreducible representations of degrees 6 and 9 defined over $\mathbb{Q}(\sqrt{-3})$. And the complex representation, associated with the action of the subgroup $\mathbb{Z}_{2} \times\left(3_{+}^{1+2} \times 3_{+}^{1+2}\right) . \mathrm{SL}_{2}(3)$, is a sum of three irreducible representations of degrees 3,3 and 9 . This decomposition gives rise to invariant sublattices of $L_{2}$, and using the general method of gluing it should be possible to obtain a computer-free description of $L_{2}$. 
Obviously, root lattices cannot be used directly to obtain lattices with minimal norm 3 or larger. But one can use a scaled copy of a root lattice as in the following example. We start with a root system over $\mathbb{Z}$, and in addition to scaling we also use the larger ground ring $\mathbb{Z}[\omega]$. Let $n$ be of the shape $n=2 r \bar{r}$ for some $r \in \mathbb{Z}[\omega]$. Let $C$ be a self-dual code over $\mathbb{F}_{4}$ of length $n$ and

$$
\Lambda=\left\langle\left(a_{1}, \ldots, a_{n}\right) \in A_{n-1} \mid\left(\varphi\left(a_{1}\right), \ldots, \varphi\left(a_{n}\right)\right) \in C\right\rangle+\frac{1}{r}\langle(1-n, 1, \ldots, 1)\rangle .
$$

Then $\frac{1}{\sqrt{2}} \Lambda$ is a unimodular hermitian lattice of rank $n-1$. This construction had been used in 2 to obtain the root-free lattice in dimension 13 . It can also be applied in e.g. dimension 17.

We close with some remarks on the theta series of our lattices. There is one interesting observation in dimension 14 which might deserve further attention.

Proposition 1. Any indecomposable unimodular lattice of rank 14, more generally any 3 -modular $\mathbb{Z}$-lattice of rank 28 with minimum $\geq 2$ has exactly $17472=2^{6} .3 .7 .13$ vectors of norm 3 .

Proof. (See 19] for the method): The relevant algebra $\mathcal{M}^{*}(3, \chi)$ of modular forms (where $\chi$ is an appropriate character) is generated by the cusp form $\Delta_{3}(q)=$ $\eta(q)^{6} \eta(3 q)^{6}$ of weight 6 and the theta-series $\theta_{3}:=\theta_{A_{2}}$ of the $A_{2}$-root lattice, of weight 1 . A linear basis for the space $\mathcal{M}_{14}(3, \chi)$ of forms of weight 14 , containing the theta-series of the lattices in question is the following:

$$
\begin{aligned}
& \theta_{3}^{14}=1+84 q+3276 q^{2}+78708 q^{3}+O\left(q^{4}\right) \\
& \theta_{3}{ }^{8} \Delta_{3}=q+42 q^{2}+729 q^{3}+O\left(q^{4}\right) \\
& \theta_{3}^{2} \Delta_{3}^{2}=q^{2}+O\left(q^{4}\right)
\end{aligned}
$$

The theta series of a lattice without vectors of norm 1 is of the form

$$
\theta_{3}^{14}-84 \theta_{3}^{8} \Delta_{3}+c \theta_{3}^{2} \Delta_{3}^{2}
$$

with $c$ given by the number of vectors of norm 2. The absence of $q^{3}$ in the third basis vector proves the claim.

Note that $M_{13} \approx 0.00000014$ (there are 14 lattices of rank 13 ), $M_{14} \approx 0.000012$ (58 lattices), $M_{15} \approx 0.0045$ (259 lattices), $M_{16} \approx 6.57, M_{17} \approx 42188.20$. So beyond dimension 15, a full classification of unimodular Eisenstein lattices may no longer be possible.

Notations in tables. For each lattice $L$ we present the sublattice $L^{(2)}$ generated by its root system, in standard notation, the number of roots $\mu_{2}(L)$ and the order $|G(L)|$ of the automorphism group. In Tables 1 and 2 we give also glue vectors from $\left(L^{(2)}\right)^{\#}$, as explained above. For instance, for lattice No. 24 the root system is $A_{6} D_{5}(2) A_{2}$, the glue vectors are given in terms of standard generators $y_{1} \in\left(A_{6}\right)^{\#}$, $y_{2} \in\left(D_{5}(2)\right)^{\#}, y_{3} \in\left(A_{2}\right)^{\#}$ and a vector $x$ of norm 84 orthogonal to $L^{(2)}$. 
Table 1. Unimodular lattices of rank 14 (obtained from self-dual codes over $\mathbb{F}_{4}$ )

\begin{tabular}{|c|c|c|c|c|}
\hline No. & $L$ & $L^{(2)}$ & $|G(L)|$ & $\mu_{2}(L)$ \\
\hline 1 & $\left\langle L^{(2)}, \frac{1}{2}(\sqrt{-3}, 1, \ldots, 1)\right\rangle$ & $D_{14}(2)$ & $2^{24} \cdot 3^{6} \cdot 5^{2} \cdot 7^{2} \cdot 11 \cdot 13$ & 1092 \\
\hline 2 & $\begin{array}{l}\left\langle L^{(2)}, y_{1}+y_{2}\right\rangle \\
y_{i} \in E_{7}^{*}-E_{7}\end{array}$ & $E_{7}^{2}$ & $2^{21} \cdot 3^{9} \cdot 5^{2} \cdot 7^{2}$ & 756 \\
\hline 3 & $\begin{array}{l}\left\langle L^{(2)}, \frac{1}{2}(\sqrt{-3}, 1, \ldots, 1)+y_{3},\right. \\
\left.\frac{1}{2}(-\sqrt{-3}, 1, \ldots, 1)+y_{2}\right\rangle \\
y_{2}=\frac{1}{2 \sqrt{-3}}(-5,1,1,1,1,1), y_{3}=\frac{1}{2}(-1,1)\end{array}$ & $D_{8}(2) U_{5} A_{1}$ & $2^{22} \cdot 3^{7} \cdot 5^{2} \cdot 7$ & 612 \\
\hline 4 & $\begin{array}{l}\left\langle L^{(2)}, y_{1}+z, y_{2}+z\right\rangle \\
z=\frac{1}{2}(\sqrt{-3}, 1,1,1) \\
y_{i}=\frac{1}{2 \sqrt{-3}}(-5,1,1,1,1,1)\end{array}$ & $U_{5}^{2} D_{4}(2)$ & $2^{21} \cdot 3^{11} \cdot 5^{2}$ & 612 \\
\hline 5 & $\begin{array}{l}\left\langle L^{(2)}\right. \\
\frac{1}{2}(1, \ldots, 1)+(1,0, \ldots, 0), \\
\left.(1,0, \ldots, 0)+\frac{1}{2}(\sqrt{-3}, 1, \ldots, 1)\right\rangle\end{array}$ & $\overline{D_{8}(2) D_{6}(2)}$ & $2^{23} \cdot 3^{5} \cdot 5^{2} \cdot 7$ & 516 \\
\hline 6 & $\begin{array}{l}\left\langle L^{(2)}\right. \\
(1,0, \ldots, 0)+(1,0, \ldots, 0)+\omega y_{1}+\omega^{2} y_{2}, \\
\frac{1}{2}(\sqrt{-3}, 1, \ldots, 1)_{1}+y_{1}+y_{2} \\
\left.\frac{1}{2}(\sqrt{-3}, 1, \ldots, 1)_{1}-\frac{1}{2}(\sqrt{-3}, 1, \ldots, 1)_{2}\right\rangle \\
y_{i}=\frac{1}{2}(-1,1)\end{array}$ & $D_{6}(2)^{2} A_{1}^{2}$ & $2^{21} \cdot 3^{5} \cdot 5^{2}$ & 372 \\
\hline 7 & $\begin{array}{l}\left\langle L^{(2)}\right. \\
(1,0, \ldots, 0)+(1,0,0,0)+(1,0,0,0) \\
(0, \ldots, 0, \omega)+\frac{1}{2}(1,1,1,1)+\frac{1}{2}(1,1,1,1) \\
\left.\frac{1}{2}(1, \ldots, 1)+\frac{1}{2}(\sqrt{-3}, 1,1,1)\right\rangle\end{array}$ & $D_{6}(2) D_{4}(2)^{2}$ & $2^{22} .3^{6} .5$ & 324 \\
\hline 8 & $\begin{array}{l}\left\langle L^{(2)}\right. \\
(1,0,0,0)_{1}+(1,0,0,0)_{2}+(1,0,0,0)_{3}, \\
y_{1}+y_{2}+y_{4}+y_{5} \\
y_{2}+y_{3}+\omega^{2} y_{4}+\omega^{2} y_{5}, \\
\left.(1,0,0,0)_{2}+\left(\omega^{2}, 0,0,0\right)_{3}+\omega y_{4}+\omega^{2} y_{5}\right\rangle \\
y_{1,2,3}=\frac{1}{2}(1,1,1,1), y_{4,5}=\frac{1}{2}(-1,1)\end{array}$ & $D_{4}(2)^{3} A_{1}^{2}$ & $2^{21} \cdot 3^{5}$ & 228 \\
\hline 9 & $\begin{array}{l}\left\langle L^{(2)}\right. \\
(1,0,0,0)_{1}+y_{3}+y_{4}+\omega y_{5}+\omega y_{6} \\
\frac{1}{2}(1,1,1,1)_{1}+\omega^{2} y_{2}+\omega^{2} y_{3}+y_{4}+y_{5} \\
(1,0,0,0)_{2}+y_{2}+\omega y_{3}+y_{5}+\omega y_{6} \\
\frac{1}{2}(1,1,1,1)_{2}+y_{2}+y_{3}+\omega^{2} y_{4}+\omega^{2} y_{5} \\
\left.y_{1}+y_{2}+y_{3}+y_{4}+y_{5}+y_{6}\right\rangle \\
y_{i}=\frac{1}{2}(-1,1)\end{array}$ & $D_{4}(2)^{2} A_{1}{ }^{6}$ & $2^{20} \cdot 3^{5}$ & 180 \\
\hline 10 & $\begin{array}{l}\left\langle L^{(2)}, \alpha_{1} y_{1}+\cdots+\alpha_{14} y_{14}\right\rangle \\
y_{i}=\frac{1}{2}(-1,1) \\
\left(\alpha_{1}, \ldots, \alpha_{14}\right) \bmod 2 \in Q R(14)\end{array}$ & $A_{1}{ }^{14}$ & $2^{16} .3^{2} .7 .13$ & 84 \\
\hline
\end{tabular}


Table 2. Unimodular lattices of rank 14

\begin{tabular}{|c|c|c|c|c|}
\hline No. & $L$ & $L^{(2)}$ & $|G(L)|$ & $\mu_{2}(L)$ \\
\hline 11 & $L_{0}$ & $\varnothing$ & $2^{8} \cdot 3^{7} \cdot 7.13$ & 0 \\
\hline 12 & $\begin{array}{l}\left\langle L^{(2)}, \frac{1}{\sqrt{-3}}(1, \ldots, 1)+y_{2}\right\rangle, \\
y_{2} \in E_{6}^{*}-E_{6}\end{array}$ & $D_{8}(\sqrt{-3}) E_{6}$ & $2^{15} \cdot 3^{14} \cdot 5^{2} \cdot 7$ & 720 \\
\hline 13 & $\begin{array}{l}\left\langle L^{(2)},(2+\sqrt{-3}) y_{1}+y_{2}\right\rangle, \\
y_{1}=\frac{1}{14}(-13,1, \ldots, 1), y_{2}=\frac{1}{2}(-1,1)\end{array}$ & $A_{13} A_{1}$ & $2^{13} \cdot 3^{6} \cdot 5^{2} \cdot 7^{2} \cdot 11 \cdot 13$ & 552 \\
\hline 14 & Complex conjugate of 13 & & & \\
\hline 15 & $\begin{array}{l}\left\langle L^{(2)},(1,0,0,0,0,0)+y_{2}+y_{3},\right. \\
\left.\frac{1}{\sqrt{-3}}(1,1,1,1,1,1)-\sqrt{-3} y_{3}\right\rangle, \\
y_{2} \in E_{6}^{*}-E_{6}, y_{3}=\frac{1}{3}(-2,1,1)\end{array}$ & $D_{6}(\sqrt{-3}) E_{6} A_{2}$ & $2^{13} \cdot 3^{13} \cdot 5^{2}$ & 504 \\
\hline 16 & $\begin{array}{l}\left\langle L^{(2)}\right. \\
\left.\frac{\sqrt{-3}}{12}(-11,1, \ldots, 1)+\frac{\sqrt{-3}}{4}(-3,1,1,1)\right\rangle\end{array}$ & $A_{11} A_{3}$ & $2^{14} \cdot 3^{7} \cdot 5^{2} \cdot 7 \cdot 11$ & 432 \\
\hline 17 & $\begin{array}{l}\left\langle L^{(2)}, \sqrt{-3} y_{1}+y_{2}\right\rangle \\
y_{1}=\frac{1}{9}(-8,1, \ldots, 1), y_{2} \in E_{6}^{*}-E_{6}\end{array}$ & $A_{8} E_{6}$ & $2^{15} \cdot 3^{9} \cdot 5^{2} .7$ & 432 \\
\hline 18 & $\begin{array}{l}\left\langle L^{(2)}, y_{1}+y_{2}+y_{3}, y_{1}-y_{2}+y_{4}\right\rangle \\
y_{1,2}=\frac{1}{\sqrt{-3}}(1, \ldots, 1), y_{3,4}=\frac{1}{3}(-2,1,1)\end{array}$ & $D_{5}(\sqrt{-3})^{2} A_{2}^{2}$ & $2^{11} \cdot 3^{13} \cdot 5^{2}$ & 396 \\
\hline 19 & $\begin{array}{l}\left\langle L^{(2)} \oplus\langle x\rangle\right. \\
\left.y_{1}+y_{2}+\frac{2}{9} x,(1,0,0,0,0)+\frac{\sqrt{-3}}{3} x\right\rangle \\
y_{1}=\frac{1}{9}(-8,1, \ldots, 1) \\
y_{2}=\frac{1}{\sqrt{-3}}(1,1,1,1,1),(x, x)=9\end{array}$ & $\overline{A_{8} D_{5}(\sqrt{-3})}$ & $2^{11} \cdot 3^{10} \cdot 5^{2} \cdot 7$ & 396 \\
\hline 20 & $\begin{array}{l}\left\langle L^{(2)},(2+\sqrt{-3}) y_{1}+y_{2}\right\rangle \\
y_{i}=\frac{1}{8}(-7,1, \ldots, 1)\end{array}$ & $A_{7}^{2}$ & $2^{15} \cdot 3^{5} \cdot 5^{2} \cdot 7^{2}$ & 336 \\
\hline 21 & $\begin{array}{l}\left\langle L^{(2)}, y_{1}+2 \sqrt{-3} y_{2}+y_{3}\right\rangle \\
y_{1}=\frac{1}{10}(-9,1, \ldots, 1) \\
y_{2}=\frac{1}{5}(-4,1, \ldots, 1), y_{3}=\frac{1}{2}(-1,1)\end{array}$ & $A_{9} A_{4} A_{1}$ & $2^{13} \cdot 3^{6} \cdot 5^{3} \cdot 7$ & 336 \\
\hline 22 & $\begin{array}{l}\left\langle L^{(2)} \oplus\langle x\rangle,\right. \\
\left.(2+\sqrt{-3}) y_{1}+\sqrt{-3} y_{2}+\frac{1}{36} x\right\rangle \\
y_{1}=\frac{1}{9}(-8,1, \ldots, 1) \\
y_{2}=\frac{1}{2}(1, \ldots, 1),(x, x)=36\end{array}$ & $A_{8} D_{5}(2)$ & $2^{15} .3^{6} \cdot 5^{2} .7$ & 336 \\
\hline 23 & Complex conjugate of 22 & & & \\
\hline 24 & $\begin{array}{l}\left\langle L^{(2)} \oplus\langle x\rangle\right. \\
\left.\sqrt{-3} y_{1}+\sqrt{-3} y_{2}+y_{3}+\frac{1}{84} x\right\rangle \\
y_{1}=\frac{1}{7}(-6,1, \ldots, 1), y_{2}=\frac{1}{\sqrt{-3}}(1, \ldots, 1) \\
y_{3}=\frac{1}{3}(-2,1,1),(x, x)=84\end{array}$ & $A_{6} D_{5}(2) A_{2}$ & $2^{13} \cdot 3^{5} \cdot 5^{2} \cdot 7$ & 264 \\
\hline 25 & $\begin{array}{l}\left\langle L^{(2)} \oplus\langle x\rangle,\right. \\
\left.y_{1}+y_{3}+\frac{2+\sqrt{-3}}{8} x, y_{2}+y_{3}+\frac{2-\sqrt{-3}}{6} x\right\rangle, \\
y_{1}=\frac{1}{8}(-7,1, \ldots, 1), y_{2}=\frac{1}{6}(-5,1, \ldots, 1), \\
y_{3}=\frac{1}{2}(-1,1),(x, x)=24\end{array}$ & $A_{7} A_{5} A_{1}$ & $2^{13} \cdot 3^{5} \cdot 5^{2} \cdot 7$ & 264 \\
\hline 26 & Complex conjugate of 25 & & & \\
\hline
\end{tabular}


Table 2. Unimodular lattices of rank 14 (continued)

\begin{tabular}{|c|c|c|c|c|}
\hline No. & $L$ & $L^{(2)}$ & $|G(L)|$ & $\mu_{2}(L)$ \\
\hline 27 & $\begin{array}{l}\left\langle L^{(2)} \oplus\langle x\rangle,\right. \\
\left.y_{1}+y_{3}+\frac{2+\sqrt{-3}}{8} x, y_{3}+y_{4}+\frac{1}{2} x, y_{2}+\frac{1}{3} x\right\rangle \\
y_{1}=\frac{1}{8}(-7,1, \ldots, 1), y_{2}=\frac{1}{\sqrt{-3}}(1,1,1,1) \\
y_{3,4}=\frac{1}{2}(-1,1),(x, x)=24\end{array}$ & $A_{7} D_{4}(\sqrt{-3}) A_{1}^{2}$ & $2^{13} \cdot 3^{7} \cdot 5 \cdot 7$ & 288 \\
\hline 28 & $\begin{array}{l}\left\langle L^{(2)}\right. \\
\left.y_{1}+y_{2}+y_{3}, 2 \sqrt{-3}\left(y_{1}-y_{2}\right)\right\rangle \\
y_{1,2}=\frac{1}{6}(-5,1, \ldots, 1), y_{3}=\frac{1}{\sqrt{-3}}(1,1,1,1)\end{array}$ & $A_{5}^{2} D_{4}(\sqrt{-3})$ & $2^{13} \cdot 3^{9} \cdot 5^{2}$ & 288 \\
\hline 29 & $\begin{array}{l}\left\langle L^{(2)} \oplus\langle x\rangle\right. \\
\left.y_{1}+\sqrt{-3} y_{2}+y_{3}+\frac{1}{60} x\right\rangle \\
y_{1}=\frac{1}{2}(1,1,1,1,1), y_{2}=\frac{1}{5}(-4,1,1,1,1) \\
y_{3}=\frac{1}{\sqrt{-3}}(1,1,1,1),(x, x)=60\end{array}$ & $D_{5}(2) A_{4} D_{4}(\sqrt{-3})$ & $2^{14} \cdot 3^{7} \cdot 5^{2}$ & 288 \\
\hline 30 & $\begin{array}{l}\left\langle L^{(2)} \oplus\langle x\rangle \oplus\langle z\rangle,\right. \\
y_{1}+\sqrt{-3} y_{2}+\frac{1}{3} x, \frac{1}{2}(\sqrt{-3}, 1,1,1)+\frac{1}{2} x, \\
\left.\sqrt{-3} y_{1}+y_{2}+\frac{1}{3} z, \frac{1}{2}(-\sqrt{-3}, 1,1,1)+\frac{1}{2} z\right\rangle, \\
y_{1,2}=\frac{1}{\sqrt{-3}}(1,1,1,1),(x, x)=6,(z, z)=6\end{array}$ & $D_{4}(\sqrt{-3})^{2} D_{4}(2)$ & $2^{14} \cdot 3^{11}$ & 288 \\
\hline 31 & $\begin{array}{l}\left\langle L^{(2)} \oplus\langle x\rangle \oplus\langle z\rangle\right. \\
\left.y_{1}+\sqrt{-3} y_{2}+\frac{2}{7} x, \sqrt{-3} y_{1}+y_{2}+\frac{2}{7} z\right\rangle \\
y_{i}=\frac{1}{7}(-6,1, \ldots, 1),(x, x)=7,(z, z)=7\end{array}$ & $A_{6}^{2}$ & $2^{10} \cdot 3^{5} \cdot 5^{2} \cdot 7^{2}$ & 252 \\
\hline 32 & $\begin{array}{l}\left\langle L^{(2)} \oplus\langle x\rangle \oplus\langle z\rangle,\right. \\
3 \sqrt{-3} y_{1}+y_{2}+\frac{1}{2}(\sqrt{-3}, 1,1,1)+\frac{1}{42} x+\frac{1}{3} z, \\
\left.\frac{1}{2}(-\sqrt{-3}, 1,1,1)+\frac{\sqrt{-3}}{3} x-\frac{\sqrt{-3}}{6} z\right\rangle \\
y_{1}=\frac{1}{7}(-6,1, \ldots, 1), y_{3}=\frac{1}{3}(-2,1,1) \\
(x, x)=42,(z, z)=6\end{array}$ & $A_{6} A_{2} D_{4}(2)$ & $2^{12} \cdot 3^{6} \cdot 5 \cdot 7$ & 216 \\
\hline 33 & $\begin{array}{l}\left\langle L^{(2)}\right. \\
\left.y_{1}+y_{2}+y_{3}+y_{4}, 2 y_{2}+\omega y_{3}-\sqrt{-3} \omega y_{4}\right\rangle \\
y_{1,2}=\frac{1}{6}(-5,1, \ldots, 1), y_{3,4}=\frac{1}{3}(-2,1,1)\end{array}$ & $A_{5}{ }^{2} A_{2}{ }^{2}$ & $2^{12} \cdot 3^{7} \cdot 5^{2}$ & 216 \\
\hline 34 & $\begin{array}{l}L^{(2)} \oplus\langle x\rangle, \\
\left.y_{1}+y_{2}+\frac{1}{3} x, y_{3}+\frac{\sqrt{-3}}{4} x, 2 \sqrt{-3}\left(y_{1}-y_{2}\right)\right\rangle \\
y_{1,2}=\frac{1}{6}(-5,1, \ldots, 1), y_{3}=\frac{1}{4}(-3,1,1,1), \\
(x, x)=12\end{array}$ & $A_{5}{ }^{2} A_{3}$ & $2^{13} \cdot 3^{6} \cdot 5^{2}$ & 216 \\
\hline 35 & Complex conjugate of 34 & & & \\
\hline 36 & $\begin{array}{l}\left\langle L^{(2)} \oplus\langle x\rangle \oplus\langle z\rangle\right. \\
2 \sqrt{-3} y_{1}+y_{2}+\frac{2}{5} x, \frac{1}{2}(\sqrt{-3}, 1,1,1)+\frac{1}{2} x \\
\left.y_{1}+2 \sqrt{-3} y_{2}+\frac{2}{5} z, \frac{1}{2}(1,-1,1, \sqrt{-3})+\frac{1}{2} z\right\rangle \\
y_{1,2}=\frac{1}{5}(-4,1, \ldots, 1) \\
(x, x)=10,(z, z)=10\end{array}$ & $\overline{A_{4}^{2} D_{4}(2)}$ & $2^{14} \cdot 3^{4} \cdot 5^{2}$ & 192 \\
\hline
\end{tabular}


Table 2. Unimodular lattices of rank 14 (continued)

\begin{tabular}{|c|c|c|c|c|}
\hline No. & $L$ & $L^{(2)}$ & $|G(L)|$ & $\mu_{2}(L)$ \\
\hline 37 & $\begin{array}{l}\left\langle L^{(2)} \oplus\langle x\rangle,\right. \\
2 y_{1}+\sqrt{-3} y_{2}+y_{3}+y_{4}+\frac{1}{60} x, \\
\left.3 \omega y_{1}+2 y_{3}+y_{4}\right\rangle,(x, x)=60 \\
y_{1}=\frac{1}{6}(-5,1, \ldots, 1), y_{3}=\frac{1}{4}(-3,1,1,1) \\
y_{2}=\frac{1}{5}(-4,1,1,1,1), y_{4}=\frac{1}{2}(-1,1)\end{array}$ & $A_{5} A_{4} A_{3} A_{1}$ & $2^{12} \cdot 3^{5} \cdot 5^{2}$ & 192 \\
\hline 38 & Complex conjugate of 37 & & & \\
\hline 39 & & $A_{5}^{2} A_{1}^{2}$ & $2^{12} \cdot 3^{5} \cdot 5^{2}$ & 192 \\
\hline 40 & & $D_{3}(\sqrt{-3})^{2} A_{2}{ }^{4}$ & $2^{10} \cdot 3^{12}$ & 180 \\
\hline 41 & & $A_{5} D_{3}(\sqrt{-3}) A_{2}^{2}$ & $2^{9} .3^{9} .5$ & 180 \\
\hline 42 & & $A_{4}^{2} D_{3}(\sqrt{-3}) A_{1}$ & $2^{10} \cdot 3^{6} \cdot 5^{2}$ & 180 \\
\hline 43 & & $D_{4}(2) D_{3}(\sqrt{-3})^{2}$ & $2^{13} \cdot 3^{10}$ & 180 \\
\hline 44 & $\begin{array}{l}\left\langle L^{(2)} \oplus\langle x\rangle \oplus\langle z\rangle\right. \\
y_{1}+2 \sqrt{-3} y_{2}+\frac{1}{5} x, y_{3}+y_{4}+\frac{1}{3} x \\
\left.2 \sqrt{-3} y_{1}+y_{2}+\frac{1}{15} z, y_{3}-y_{4}+\frac{1}{3} z\right\rangle \\
y_{1,2}=\frac{1}{5}(-4,1, \ldots, 1),(x, x)=15 \\
y_{3,4}=\frac{1}{3}(-2,1,1),(z, z)=15\end{array}$ & $A_{4}^{2} A_{2}^{2}$ & $2^{10} \cdot 3^{5} \cdot 5^{2}$ & 156 \\
\hline 45 & $\begin{array}{l}\left\langle L^{(2)} \oplus\langle x\rangle \oplus\langle z\rangle \oplus\langle u\rangle,\right. \\
2 \sqrt{-3}\left(y_{1}-y_{2}\right)+y_{3}+\frac{1}{2} u+\frac{1}{20} x, \\
\left.2 \sqrt{-3}\left(y_{1}+y_{2}\right)+2 y_{3}+\frac{\sqrt{-3}}{4} u+\frac{1}{20} z\right\rangle, \\
y_{1,2}=\frac{1}{5}(-4,1, \ldots, 1),(x, x)=20, \\
y_{3}=\frac{1}{4}(-3,1,1,1),(z, z)=20,(u, u)=4\end{array}$ & $A_{4}^{2} A_{3}$ & $2^{11} \cdot 3^{4} \cdot 5^{2}$ & 156 \\
\hline 46 & & $A_{4} A_{3}^{2} A_{1}^{2}$ & $2^{13} \cdot 3^{4} \cdot 5$ & 144 \\
\hline 47 & & $A_{3}{ }^{4}$ & $2^{15} \cdot 3^{5}$ & 144 \\
\hline 48 & & $A_{3}^{2} A_{2}^{2} A_{1}^{2}$ & $2^{12} \cdot 3^{5}$ & 120 \\
\hline 49 & & $A_{3}^{3} A_{1}^{2}$ & $2^{13} \cdot 3^{5}$ & 120 \\
\hline 50 & Complex conjugate of 49 & & & \\
\hline 51 & & $A_{2}{ }^{6}$ & $2^{9} \cdot 3^{9}$ & 108 \\
\hline 52 & & $A_{3} A_{2}{ }^{4}$ & $2^{10} \cdot 3^{7}$ & 108 \\
\hline 53 & & $A_{3}{ }^{2} A_{2}{ }^{2}$ & $2^{11} \cdot 3^{6}$ & 108 \\
\hline 54 & & $A_{2}{ }^{4} A_{1}{ }^{2}$ & $2^{10} \cdot 3^{5}$ & 84 \\
\hline 55 & & $A_{2}^{2} A_{1}{ }^{6}$ & $2^{11} \cdot 3^{5}$ & 72 \\
\hline 56 & & $A_{2}{ }^{4}$ & $2^{8} .3^{9}$ & 72 \\
\hline 57 & & $A_{1}^{8}$ & $2^{13} \cdot 3^{2} \cdot 7$ & 48 \\
\hline 58 & & $A_{1}{ }^{6}$ & $2^{13} \cdot 3^{4} .5$ & 36 \\
\hline
\end{tabular}


Table 3. Unimodular lattices of rank 15

\begin{tabular}{|c|c|c|c|}
\hline No. & $L^{(2)}$ & $|G(L)|$ & $\mu_{2}(L)$ \\
\hline 1 & $\varnothing$ & $2^{9} .3^{7} .5 .7$ & 0 \\
\hline 2 & $\varnothing$ & $2^{5} \cdot 3^{7}$ & 0 \\
\hline 3 & $D_{15}(\sqrt{-3})$ & $2^{12} \cdot 3^{20} \cdot 5^{3} \cdot 7^{2} \cdot 11.13$ & 1890 \\
\hline 4 & $D_{9}(\sqrt{-3}) D_{6}(\sqrt{-3})$ & $2^{12} \cdot 3^{19} \cdot 5^{2} .7$ & 918 \\
\hline 5 & $E_{7} D_{7}(\sqrt{-3})$ & $2^{15} \cdot 3^{13} \cdot 5^{2} \cdot 7^{2}$ & 756 \\
\hline 6 & $A_{15}$ & $2^{16} \cdot 3^{7} \cdot 5^{3} \cdot 7^{2} \cdot 11 \cdot 13$ & 720 \\
\hline 7 & $A_{14}$ & $2^{12} \cdot 3^{7} \cdot 5^{3} \cdot 7^{2} \cdot 11 \cdot 13$ & 630 \\
\hline 8 & complex conjugate of 7 & & \\
\hline 9 & $D_{9}(2) D_{5}(\sqrt{-3})$ & $2^{19} \cdot 3^{10} \cdot 5^{2} \cdot 7$ & 612 \\
\hline 10 & $A_{8} D_{7}(\sqrt{-3})$ & $2^{12} \cdot 3^{13} \cdot 5^{2} \cdot 7^{2}$ & 594 \\
\hline 11 & $D_{6}(\sqrt{-3}) D_{6}(\sqrt{-3}) D_{3}(\sqrt{-3})$ & $2^{11} \cdot 3^{17} \cdot 5^{2}$ & 594 \\
\hline 12 & $A_{9} U_{5}$ & $2^{16} \cdot 3^{9} \cdot 5^{3} .7$ & 540 \\
\hline 13 & $A_{5} D_{5}(\sqrt{-3}) U_{5}$ & $2^{15} \cdot 3^{12} \cdot 5^{3}$ & 540 \\
\hline 14 & $E_{6} U_{5} D_{3}(\sqrt{-3})$ & $2^{16} \cdot 3^{13} \cdot 5^{2}$ & 540 \\
\hline 15 & $A_{11} D_{4}(\sqrt{-3})$ & $2^{14} \cdot 3^{10} \cdot 5^{2} \cdot 7.11$ & 504 \\
\hline 16 & $A_{8} D_{6}(\sqrt{-3})$ & $2^{12} \cdot 3^{12} \cdot 5^{2} \cdot 7$ & 486 \\
\hline 17 & $D_{7}(2) E_{6}$ & $2^{18} .3^{8} .5^{2} .7$ & 468 \\
\hline 18 & $A_{11} D_{3}(\sqrt{-3})$ & $2^{12} \cdot 3^{9} \cdot 5^{2} \cdot 7.11$ & 450 \\
\hline 19 & $A_{9} D_{5}(\sqrt{-3})$ & $2^{12} \cdot 3^{10} \cdot 5^{3} \cdot 7$ & 450 \\
\hline 20 & complex conjugate of 20 & & \\
\hline 21 & $A_{8} E_{6}$ & $2^{15} \cdot 3^{9} .5^{2} .7$ & 432 \\
\hline 22 & $D_{6}(\sqrt{-3}) D_{3}^{3}(\sqrt{-3})$ & $2^{9} .3^{18} .5$ & 432 \\
\hline 23 & $U_{5} D_{4}(\sqrt{-3}) D_{4}(\sqrt{-3})$ & $2^{15} \cdot 3^{13} .5$ & 432 \\
\hline 24 & $D_{7}(2) A_{7}$ & $2^{18} \cdot 3^{5} \cdot 5^{2} \cdot 7^{2}$ & 420 \\
\hline 25 & $D_{6}(2) D_{5}(\sqrt{-3}) A_{3}$ & $2^{16} \cdot 3^{9} \cdot 5^{2}$ & 396 \\
\hline 26 & $A_{10} A_{4}$ & $2^{12} \cdot 3^{6} \cdot 5^{3} \cdot 7 \cdot 11$ & 390 \\
\hline 27 & $A_{5} D_{5}(\sqrt{-3}) D_{4}(\sqrt{-3})$ & $2^{11} \cdot 3^{12} \cdot 5^{2}$ & 378 \\
\hline 28 & $A_{9} A_{5}$ & $2^{13} \cdot 3^{7} \cdot 5^{3} \cdot 7$ & 360 \\
\hline 29 & complex conjugate of 28 & & \\
\hline 30 & $A_{7} D_{6}(2)$ & $2^{17} \cdot 3^{5} \cdot 5^{2} .7$ & 348 \\
\hline 31 & $A_{6} D_{5}(\sqrt{-3}) A_{3}$ & $2^{11} \cdot 3^{9} \cdot 5^{2} \cdot 7$ & 342 \\
\hline 32 & $A_{8} A_{6}$ & $2^{12} \cdot 3^{7} \cdot 5^{2} \cdot 7^{2}$ & 342 \\
\hline 33 & complex conjugate of 32 & & \\
\hline 34 & $A_{8} D_{4}(\sqrt{-3}) A_{2}$ & $2^{12} \cdot 3^{10} .5 .7$ & 342 \\
\hline 35 & complex conjugate of 34 & & \\
\hline 36 & $A_{8} D_{5}(2)$ & $2^{15} \cdot 3^{6} .5^{2} .7$ & 336 \\
\hline 37 & $D_{5}(\sqrt{-3}) D_{4}(2) D_{4}(2)$ & $2^{17} \cdot 3^{10} \cdot 5$ & 324 \\
\hline 38 & $D_{6}(2) A_{5} D_{3}(\sqrt{-3})$ & $2^{15} \cdot 3^{8} \cdot 5^{2}$ & 324 \\
\hline 39 & $A_{8} D_{3}(\sqrt{-3}) A_{3}$ & $2^{12} \cdot 3^{9} \cdot 5.7$ & 306 \\
\hline 40 & $A_{8} A_{5}$ & $2^{12} \cdot 3^{7} .5^{2} .7$ & 306 \\
\hline 41 & complex conjugate of 40 & & \\
\hline 42 & $A_{7} A_{6}$ & $2^{12} \cdot 3^{5} \cdot 5^{2} \cdot 7^{2}$ & 294 \\
\hline 43 & complex conjugate of 42 & & \\
\hline
\end{tabular}


Table 3. Unimodular lattices of rank 15 (continued)

\begin{tabular}{|c|c|c|c|}
\hline No. & $L^{(2)}$ & $|G(L)|$ & $\mu_{2}(L)$ \\
\hline 44 & $D_{5}(2) D_{4}(\sqrt{-3}) A_{4}$ & $2^{14} \cdot 3^{7} \cdot 5^{2}$ & 288 \\
\hline 45 & $D_{4}(\sqrt{-3}) A_{5} A_{5}$ & $2^{12} \cdot 3^{9} \cdot 5^{2}$ & 288 \\
\hline 46 & $D_{5}(2) D_{5}(2) A_{3}$ & $2^{19} \cdot 3^{4} \cdot 5^{2}$ & 276 \\
\hline 47 & $A_{6} A_{5} D_{3}(\sqrt{-3})$ & $2^{10} .3^{8} \cdot 5^{2} .7$ & 270 \\
\hline 48 & $A_{6} D_{4}(\sqrt{-3}) A_{3}$ & $2^{11} \cdot 3^{8} \cdot 5.7$ & 270 \\
\hline 49 & $A_{5} D_{4}(\sqrt{-3}) D_{3}(\sqrt{-3}) A_{2}$ & $2^{10} \cdot 3^{11} .5$ & 270 \\
\hline 50 & $D_{4}(\sqrt{-3}) D_{4}(\sqrt{-3}) A_{2} A_{2} A_{2}$ & $2^{11} \cdot 3^{13}$ & 270 \\
\hline 51 & $D_{3}(\sqrt{-3})^{5}$ & $2^{8} .3^{16} .5$ & 270 \\
\hline 52 & $A_{6} A_{6} A_{2}$ & $2^{11} \cdot 3^{6} \cdot 5^{2} \cdot 7^{2}$ & 270 \\
\hline 53 & complex conjugate of 52 & & \\
\hline 54 & $A_{7} A_{4} A_{3}$ & $2^{14} \cdot 3^{5} \cdot 5^{2} \cdot 7$ & 264 \\
\hline 55 & $A_{6} D_{5}(2) A_{2}$ & $2^{13} \cdot 3^{5} \cdot 5^{2} \cdot 7$ & 264 \\
\hline 56 & complex conjugate of 55 & & \\
\hline 57 & $A_{6} A_{6} A_{1}$ & $2^{10} \cdot 3^{5} \cdot 5^{2} \cdot 7^{2}$ & 258 \\
\hline 58 & $D_{5}(2) D_{4}(2) D_{3}(\sqrt{-3}) A_{1}$ & $2^{16} .3^{7} .5$ & 252 \\
\hline 59 & $A_{5} A_{5} D_{4}(2)$ & $2^{16} \cdot 3^{7} \cdot 5^{2}$ & 252 \\
\hline 60 & $A_{7} A_{4} A_{2}$ & $2^{12} \cdot 3^{5} \cdot 5^{2} .7$ & 246 \\
\hline 61 & $D_{5}(2) A_{4} A_{4}$ & $2^{14} \cdot 3^{4} \cdot 5^{3}$ & 240 \\
\hline 62 & $D_{5}(2) A_{4} A_{4}$ & $2^{15} \cdot 3^{4} \cdot 5^{3}$ & 240 \\
\hline 63 & $A_{7} A_{3} A_{3}$ & $2^{15} \cdot 3^{5} \cdot 5.7$ & 240 \\
\hline 64 & $A_{5} D_{4}(\sqrt{-3}) A_{2} A_{2}$ & $2^{10} .3^{9} .5$ & 234 \\
\hline 65 & $A_{4} A_{4} D_{4}(\sqrt{-3}) A_{1}$ & $2^{11} \cdot 3^{7} \cdot 5^{2}$ & 234 \\
\hline 66 & $A_{6} A_{5} A_{2}$ & $2^{10} .3^{6} \cdot 5^{2} .7$ & 234 \\
\hline 67 & $A_{6} A_{5} A_{2}$ & $2^{10} .3^{6} .5^{2} .7$ & 234 \\
\hline 68 & complex conjugate of 67 & & \\
\hline 69 & $A_{5} A_{5} D_{3}(\sqrt{-3})$ & $2^{11} \cdot 3^{8} \cdot 5^{2}$ & 234 \\
\hline 70 & complex conjugate of 69 & & \\
\hline 71 & $D_{5}(2) A_{3} A_{3} A_{3}$ & $2^{17} \cdot 3^{6} .5$ & 228 \\
\hline 72 & $A_{6} A_{4} A_{3}$ & $2^{11} \cdot 3^{5} \cdot 5^{2} .7$ & 222 \\
\hline 73 & complex conjugate of 72 & & \\
\hline 74 & $D_{4}(\sqrt{-3}) A_{3} A_{3} A_{3}$ & $2^{14} \cdot 3^{9}$ & 216 \\
\hline 75 & $D_{4}(2) D_{4}(\sqrt{-3}) A_{2} A_{2}$ & $2^{13} \cdot 3^{10}$ & 216 \\
\hline 76 & $D_{4}(\sqrt{-3}) D_{3}(\sqrt{-3}) A_{2} A_{2} A_{2}$ & $2^{9} \cdot 3^{13}$ & 216 \\
\hline 77 & $A_{5} D_{3}(\sqrt{-3})^{2} A_{2}$ & $2^{9} \cdot 3^{11} .5$ & 216 \\
\hline 78 & $A_{6} D_{3}(\sqrt{-3}) A_{2} A_{2}$ & $2^{9} \cdot 3^{8} \cdot 5.7$ & 216 \\
\hline 79 & $A_{6} D_{4}(2) A_{2}$ & $2^{12} \cdot 3^{6} \cdot 5.7$ & 216 \\
\hline 80 & $A_{5} A_{5} A_{3}$ & $2^{12} \cdot 3^{6} \cdot 5^{2}$ & 216 \\
\hline 81 & $A_{5} A_{5} A_{3}$ & $2^{13} \cdot 3^{6} \cdot 5^{2}$ & 216 \\
\hline 82 & complex conjugate of 81 & & \\
\hline 83 & $A_{6} A_{4} A_{2} A_{1}$ & $2^{10} .3^{5} \cdot 5^{2} .7$ & 210 \\
\hline 84 & complex conjugate of 83 & & \\
\hline 85 & $A_{5} A_{4} A_{4}$ & $2^{11} \cdot 3^{5} \cdot 5^{3}$ & 210 \\
\hline 86 & complex conjugate of 85 & & \\
\hline 87 & $A_{5} D_{4}(2) A_{3} A_{1}$ & $2^{15} \cdot 3^{5} \cdot 5$ & 204 \\
\hline
\end{tabular}


Table 3. Unimodular lattices of rank 15 (continued)

\begin{tabular}{|c|c|c|c|}
\hline No. & $L^{(2)}$ & $|G(L)|$ & $\mu_{2}(L)$ \\
\hline 88 & $A_{6} A_{3} A_{2} A_{2}$ & $2^{10} .3^{6} \cdot 5.7$ & 198 \\
\hline 89 & $A_{5} A_{5} A_{2}$ & $2^{11} \cdot 3^{7} .5^{2}$ & 198 \\
\hline 90 & $A_{5} A_{5} A_{2}$ & $2^{11} \cdot 3^{6} \cdot 5^{2}$ & 198 \\
\hline 91 & complex conjugate of 90 & & \\
\hline 92 & $A_{5} A_{3} D_{3}(\sqrt{-3}) A_{2}$ & $2^{10} .3^{8} .5$ & 198 \\
\hline 93 & complex conjugate of 92 & & \\
\hline 94 & $A_{5} A_{4} A_{3} A_{1}$ & $2^{12} \cdot 3^{5} \cdot 5^{2}$ & 192 \\
\hline 95 & complex conjugate of 94 & & \\
\hline 96 & $A_{4} A_{4} D_{4}(2)$ & $2^{14} \cdot 3^{4} \cdot 5^{2}$ & 192 \\
\hline 97 & complex conjugate of 96 & & \\
\hline 98 & $A_{5} A_{4} A_{3}$ & $2^{11} \cdot 3^{5} \cdot 5^{2}$ & 186 \\
\hline 99 & $A_{5} A_{4} A_{3}$ & $2^{11} \cdot 3^{5} \cdot 5^{2}$ & 186 \\
\hline 100 & $A_{5} D_{4}(2) A_{1} A_{1} A_{1}$ & $2^{14} \cdot 3^{6} .5$ & 180 \\
\hline 101 & $D_{4}(2) D_{3}(\sqrt{-3}) A_{3} A_{1} A_{1} A_{1}$ & $2^{14} \cdot 3^{7}$ & 180 \\
\hline 102 & $D_{4} D_{4}(2) A_{3}$ & $2^{17} \cdot 3^{6}$ & 180 \\
\hline 103 & $A_{3} A_{3} A_{3} A_{3} A_{3}$ & $2^{18} \cdot 3^{7} \cdot 5$ & 180 \\
\hline 104 & $A_{4} A_{4} A_{4}$ & $2^{10} \cdot 3^{5} \cdot 5^{3}$ & 180 \\
\hline 105 & complex conjugate of 104 & & \\
\hline 106 & $A_{5} A_{4} A_{2} A_{1}$ & $2^{10} \cdot 3^{5} \cdot 5^{2}$ & 174 \\
\hline 107 & $A_{5} A_{4} A_{2} A_{1}$ & $2^{10} \cdot 3^{5} \cdot 5^{2}$ & 174 \\
\hline 108 & complex conjugate of 107 & & \\
\hline 109 & $A_{4} A_{4} A_{3} A_{2}$ & $2^{11} \cdot 3^{5} \cdot 5^{2}$ & 174 \\
\hline 110 & complex conjugate of 109 & & \\
\hline 111 & $A_{4} D_{4}(2) A_{2} A_{2}$ & $2^{12} \cdot 3^{5} .5$ & 168 \\
\hline 112 & $A_{4} D_{4}(2) A_{2} A_{2}$ & $2^{13} \cdot 3^{5} .5$ & 168 \\
\hline 113 & $A_{4} A_{3} A_{3} A_{3}$ & $2^{14} \cdot 3^{5} .5$ & 168 \\
\hline 114 & $A_{5} A_{3} A_{3} A_{1}$ & $2^{13} \cdot 3^{5} .5$ & 168 \\
\hline 115 & complex conjugate of 114 & & \\
\hline 116 & $A_{4} D_{3}(\sqrt{-3}) A_{3} A_{1} A_{1}$ & $2^{10} .3^{6} .5$ & 162 \\
\hline 117 & $A_{3} A_{3} A_{3} D_{3}(\sqrt{-3})$ & $2^{11} \cdot 3^{8}$ & 162 \\
\hline 118 & $A_{3} A_{3} D_{3}(\sqrt{-3}) A_{2} A_{2}$ & $2^{11} \cdot 3^{8}$ & 162 \\
\hline 119 & $A_{5} A_{2} A_{2} A_{2} A_{2}$ & $2^{9} .3^{8} .5$ & 162 \\
\hline 120 & $D_{3}(\sqrt{-3}) D_{3}(\sqrt{-3}) A_{2} A_{2} A_{2}$ & $2^{7} \cdot 3^{12}$ & 162 \\
\hline 121 & $A_{5} A_{3} A_{2} A_{2}$ & $2^{10} .3^{6} .5$ & 162 \\
\hline 122 & complex conjugate of 121 & & \\
\hline 123 & $A_{4} A_{4} A_{3} A_{1}$ & $2^{11} \cdot 3^{4} \cdot 5^{2}$ & 162 \\
\hline 124 & complex conjugate of 123 & & \\
\hline 125 & $A_{4} A_{4} A_{2} A_{2}$ & $2^{10} \cdot 3^{5} \cdot 5^{2}$ & 156 \\
\hline 126 & $D_{4}(2) A_{3} A_{3} A_{1} A_{1}$ & $2^{16} \cdot 3^{4}$ & 156 \\
\hline 127 & $A_{4} A_{4} A_{2} A_{1} A_{1}$ & $2^{10} \cdot 3^{4} \cdot 5^{2}$ & 150 \\
\hline 128 & $A_{4} A_{4} A_{2} A_{1} A_{1}$ & $2^{11} \cdot 3^{4} \cdot 5^{2}$ & 150 \\
\hline 129 & $A_{4} A_{3} A_{3} A_{2}$ & $2^{11} \cdot 3^{5} .5$ & 150 \\
\hline 130 & $A_{4} A_{3} A_{3} A_{2}$ & $2^{11} \cdot 3^{5} .5$ & 150 \\
\hline 131 & $A_{4} A_{3} A_{3} A_{2}$ & $2^{11} \cdot 3^{5} \cdot 5$ & 150 \\
\hline 132 & complex conjugate of 131 & & \\
\hline
\end{tabular}


Table 3. Unimodular lattices of rank 15 (continued)

\begin{tabular}{|c|c|c|c|}
\hline No. & $L^{(2)}$ & $|G(L)|$ & $\mu_{2}(L)$ \\
\hline 133 & $A_{4} A_{3} A_{3} A_{1} A_{1}$ & $2^{12} \cdot 3^{4} .5$ & 144 \\
\hline 134 & $A_{3} D_{3}(\sqrt{-3}) A_{3} A_{2} A_{2} A_{2}$ & $2^{8} \cdot 3^{9}$ & 144 \\
\hline 135 & $A_{5} A_{2} A_{2} A_{1} A_{1} A_{1}$ & $2^{11} \cdot 3^{6} .5$ & 144 \\
\hline 136 & $A_{4} D_{3}(\sqrt{-3}) A_{2} A_{1} A_{1}$ & $2^{9} .3^{7} .5$ & 144 \\
\hline 137 & $A_{5} A_{2} A_{2} A_{2}$ & $2^{9} .3^{8} .5$ & 144 \\
\hline 138 & $A_{3} A_{3} A_{3} A_{3}$ & $2^{16} \cdot 3^{6}$ & 144 \\
\hline 139 & $D_{4}(2) A_{2} A_{2} A_{2} A_{2}$ & $2^{12} \cdot 3^{8}$ & 144 \\
\hline 140 & $D_{4}(2) A_{2} A_{2} A_{2} A_{2}$ & $2^{13} \cdot 3^{7}$ & 144 \\
\hline 141 & $A_{3} A_{3} A_{3} A_{2} A_{2}$ & $2^{13} \cdot 3^{7}$ & 144 \\
\hline 142 & complex conjugate of 141 & & \\
\hline 143 & $A_{4} A_{4} A_{1} A_{1} A_{1}$ & $2^{11} \cdot 3^{3} \cdot 5^{2}$ & 138 \\
\hline 144 & $A_{4} A_{3} A_{2} A_{2} A_{1}$ & $2^{10} .3^{5} .5$ & 138 \\
\hline 145 & $A_{4} A_{3} A_{2} A_{2} A_{1}$ & $2^{10} .3^{5} .5$ & 138 \\
\hline 146 & complex conjugate of 145 & & \\
\hline 147 & $A_{4} A_{3} A_{2} A_{2} A_{1}$ & $2^{10} \cdot 3^{5} .5$ & 138 \\
\hline 148 & complex conjugate of 147 & & \\
\hline 149 & $A_{4} A_{3} A_{3} A_{1}$ & $2^{11} \cdot 3^{4} .5$ & 138 \\
\hline 150 & complex conjugate of 149 & & \\
\hline 151 & $A_{4} A_{3} A_{2} A_{2}$ & $2^{9} .3^{5} .5$ & 132 \\
\hline 152 & $A_{3} D_{3}(\sqrt{-3}) A_{2} A_{1} A_{1} A_{1}$ & $2^{9} .3^{7}$ & 126 \\
\hline 153 & $D_{3}(\sqrt{-3}) A_{2} A_{2} A_{2} A_{2}$ & $2^{8} .3^{9}$ & 126 \\
\hline 154 & $A_{3} A_{3} A_{3} A_{2}$ & $2^{11} \cdot 3^{6}$ & 126 \\
\hline 155 & $A_{3} A_{3} A_{2} A_{2} A_{2}$ & $2^{11} \cdot 3^{6}$ & 126 \\
\hline 156 & $A_{3} A_{3} A_{2} A_{2} A_{2}$ & $2^{11} \cdot 3^{6}$ & 126 \\
\hline 157 & complex conjugate of 156 & & \\
\hline 158 & $A_{3} A_{3} A_{2} A_{2} A_{2}$ & $2^{11} \cdot 3^{7}$ & 126 \\
\hline 159 & complex conjugate of 158 & & \\
\hline 160 & $A_{4} A_{3} A_{2} A_{1} A_{1}$ & $2^{10} .3^{4} .5$ & 126 \\
\hline 161 & complex conjugate of 160 & & \\
\hline 162 & $A_{3} A_{3} A_{2} A_{2} A_{1} A_{1}$ & $2^{11} \cdot 3^{5}$ & 120 \\
\hline 163 & $A_{4} A_{3} A_{1} A_{1} A_{1} A_{1}$ & $2^{12} \cdot 3^{3} \cdot 5$ & 120 \\
\hline 164 & $A_{4} A_{3} A_{3} A_{3} A_{1}$ & $2^{8} .3^{6} .5$ & 120 \\
\hline 165 & $A_{3} A_{3} A_{3} A_{1} A_{1}$ & $2^{12} \cdot 3^{5}$ & 120 \\
\hline 166 & $A_{3} A_{3} A_{3} A_{1} A_{1}$ & $2^{13} \cdot 3^{4}$ & 120 \\
\hline 167 & complex conjugate of 166 & & \\
\hline 168 & $A_{4} A_{2} A_{2} A_{1} A_{1} A_{1}$ & $2^{9} .3^{4} .5$ & 114 \\
\hline 169 & $A_{3} A_{3} A_{2} A_{2} A_{1}$ & $2^{10} \cdot 3^{5}$ & 114 \\
\hline 170 & $A_{3} A_{3} A_{2} A_{2} A_{1}$ & $2^{10} \cdot 3^{5}$ & 114 \\
\hline 171 & complex conjugate of 170 & & \\
\hline 172 & $A_{3} A_{3} A_{2} A_{2} A_{1}$ & $2^{11} \cdot 3^{5}$ & 114 \\
\hline 173 & complex conjugate of 172 & & \\
\hline
\end{tabular}


Table 3. Unimodular lattices of rank 15 (continued)

\begin{tabular}{|c|c|c|c|}
\hline No. & $L^{(2)}$ & $|G(L)|$ & $\mu_{2}(L)$ \\
\hline 174 & $A_{4} A_{2} A_{2} A_{1} A_{1}$ & $2^{9} \cdot 3^{5} .5$ & 108 \\
\hline 175 & $A_{3} A_{3} A_{2} A_{2}$ & $2^{10} \cdot 3^{6}$ & 108 \\
\hline 176 & $A_{3} A_{2} A_{2} A_{2} A_{2}$ & $2^{9} .3^{6}$ & 108 \\
\hline 177 & $A_{3} A_{3} A_{1} A_{1} A_{1} A_{1} A_{1} A_{1}$ & $2^{14} \cdot 3^{4}$ & 108 \\
\hline 178 & $D_{3}(\sqrt{-3}) A_{2} A_{2} A_{2}$ & $2^{6} \cdot 3^{11}$ & 108 \\
\hline 179 & $D_{3}(\sqrt{-3}) D_{3}(\sqrt{-3})$ & $2^{10} \cdot 3^{13}$ & 108 \\
\hline 180 & $D_{3}(\sqrt{-3}) A_{1} A_{1} A_{1} A_{1} A_{1} A_{1} A_{1} A_{1} A_{1}$ & $2^{14} \cdot 3^{7}$ & 108 \\
\hline 181 & $A_{2} A_{2} A_{2} A_{2} A_{2} A_{2}$ & $2^{8} .3^{9}$ & 108 \\
\hline 182 & $A_{2} A_{2} A_{2} A_{2} A_{2} A_{2}$ & $2^{10} \cdot 3^{10}$ & 108 \\
\hline 183 & $A_{3} A_{3} A_{2} A_{1} A_{1}$ & $2^{10} \cdot 3^{4}$ & 102 \\
\hline 184 & $A_{3} A_{2} A_{2} A_{2} A_{1} A_{1}$ & $2^{9} \cdot 3^{5}$ & 102 \\
\hline 185 & $A_{3} A_{2} A_{2} A_{2} A_{1} A_{1}$ & $2^{9} \cdot 3^{5}$ & 102 \\
\hline 186 & $A_{3} A_{2} A_{2} A_{2} A_{1} A_{1}$ & $2^{9} \cdot 3^{5}$ & 102 \\
\hline 187 & complex conjugate of 186 & & \\
\hline 188 & $A_{3} A_{3} A_{2} A_{1} A_{1}$ & $2^{11} \cdot 3^{4}$ & 102 \\
\hline 189 & complex conjugate of 188 & & \\
\hline 190 & $A_{3} A_{3} A_{2} A_{1} A_{1}$ & $2^{11} \cdot 3^{4}$ & 102 \\
\hline 191 & complex conjugate of 190 & & \\
\hline 192 & $A_{3} A_{2} A_{2} A_{1} A_{1} A_{1} A_{1}$ & $2^{11} \cdot 3^{4}$ & 96 \\
\hline 193 & $A_{3} A_{3} A_{1} A_{1} A_{1} A_{1}$ & $2^{13} \cdot 3^{3}$ & 96 \\
\hline 194 & $A_{3} A_{2} A_{2} A_{2} A_{1}$ & $2^{9} .3^{5}$ & 96 \\
\hline 195 & $A_{3} A_{2} A_{2} A_{1} A_{1} A_{1} A_{1}$ & $2^{12} \cdot 3^{4}$ & 96 \\
\hline 196 & $A_{3} A_{3} A_{1} A_{1} A_{1}$ & $2^{11} \cdot 3^{4}$ & 90 \\
\hline 197 & $A_{2} A_{2} A_{2} A_{2} A_{1} A_{1} A_{1}$ & $2^{9} \cdot 3^{6}$ & 90 \\
\hline 198 & $A_{3} A_{2} A_{2} A_{1} A_{1} A_{1}$ & $2^{9} .3^{5}$ & 90 \\
\hline 199 & $A_{3} A_{2} A_{2} A_{1} A_{1} A_{1}$ & $2^{9} \cdot 3^{4}$ & 90 \\
\hline 200 & $A_{3} A_{2} A_{2} A_{1} A_{1} A_{1}$ & $2^{9} .3^{4}$ & 90 \\
\hline 201 & complex conjugate of 200 & & \\
\hline 202 & $A_{2} A_{2} A_{2} A_{2} A_{2}$ & $2^{8} \cdot 3^{7}$ & 90 \\
\hline 203 & $A_{2} A_{2} A_{2} A_{2} A_{2}$ & $2^{7} .3^{7}$ & 90 \\
\hline 204 & complex conjugate of 203 & & \\
\hline 205 & $A_{2} A_{2} A_{2} A_{2} A_{2}$ & $2^{8} .3^{7} .5$ & 90 \\
\hline 206 & complex conjugate of 205 & & \\
\hline 207 & $A_{3} A_{2} A_{2} A_{1} A_{1}$ & $2^{9} \cdot 3^{4}$ & 84 \\
\hline 208 & $A_{3} A_{1} A_{1} A_{1} A_{1} A_{1} A_{1} A_{1} A_{1}$ & $2^{15} \cdot 3^{3}$ & 84 \\
\hline 209 & $A_{2} A_{2} A_{2} A_{2} A_{1} A_{1}$ & $2^{8} \cdot 3^{5}$ & 84 \\
\hline 210 & complex conjugate of 209 & & \\
\hline 211 & $A_{3} A_{2} A_{1} A_{1} A_{1} A_{1}$ & $2^{9} \cdot 3^{3}$ & 78 \\
\hline 212 & $A_{2} A_{2} A_{2} A_{2} A_{1}$ & $2^{7} \cdot 3^{5}$ & 78 \\
\hline 213 & $A_{2} A_{2} A_{2} A_{1} A_{1} A_{1} A_{1}$ & $2^{9} \cdot 3^{4}$ & 78 \\
\hline 214 & $A_{2} A_{2} A_{2} A_{1} A_{1} A_{1} A_{1}$ & $2^{9} .3^{4}$ & 78 \\
\hline 215 & complex conjugate of 214 & & \\
\hline
\end{tabular}


Table 3. Unimodular lattices of rank 15 (continued)

\begin{tabular}{|c|c|c|c|}
\hline No. & $L^{(2)}$ & $|G(L)|$ & $\mu_{2}(L)$ \\
\hline 216 & $A_{3} A_{2} A_{2}$ & $2^{8} \cdot 3^{6}$ & 72 \\
\hline 217 & $A_{3} A_{2} A_{1} A_{1} A_{1}$ & $2^{9} \cdot 3^{5}$ & 72 \\
\hline 218 & $A_{2} A_{2} A_{2} A_{1} A_{1} A_{1}$ & $2^{7} \cdot 3^{5}$ & 72 \\
\hline 219 & $A_{3} A_{3} A_{3} A_{1} A_{1} A_{1}$ & $2^{8} \cdot 3^{6}$ & 72 \\
\hline 220 & $A_{2} A_{2} A_{1} A_{1} A_{1} A_{1} A_{1} A_{1}$ & $2^{10} \cdot 3^{5}$ & 72 \\
\hline 221 & $A_{2} A_{2} A_{1} A_{1} A_{1} A_{1} A_{1} A_{1}$ & $2^{10} \cdot 3^{4}$ & 72 \\
\hline 222 & complex conjugate of 221 & & \\
\hline 223 & $A_{3} A_{1} A_{1} A_{1} A_{1} A_{1} A_{1}$ & $2^{12} \cdot 3^{3}$ & 72 \\
\hline 224 & complex conjugate of 223 & & \\
\hline 225 & $A_{2} A_{2} A_{2} A_{1} A_{1}$ & $2^{7} \cdot 3^{4}$ & 66 \\
\hline 226 & $A_{2} A_{2} A_{2} A_{1} A_{1}$ & $2^{7} \cdot 3^{5}$ & 66 \\
\hline 227 & $A_{2} A_{2} A_{1} A_{1} A_{1} A_{1} A_{1}$ & $2^{8} \cdot 3^{3}$ & 66 \\
\hline 228 & $A_{2} A_{2} A_{1} A_{1} A_{1} A_{1} A_{1}$ & $2^{9} \cdot 3^{3}$ & 66 \\
\hline 229 & complex conjugate of 228 & & \\
\hline 230 & $A_{2} A_{2} A_{1} A_{1} A_{1} A_{1}$ & $2^{8} \cdot 3^{3}$ & 60 \\
\hline 231 & $A_{2} A_{2} A_{1} A_{1} A_{1} A_{1}$ & $2^{10} .3^{3}$ & 60 \\
\hline 232 & $A_{1} A_{1} A_{1} A_{1} A_{1} A_{1} A_{1} A_{1} A_{1} A_{1}$ & $2^{13} \cdot 3^{2} .5$ & 60 \\
\hline 233 & $A_{2} A_{2} A_{2}$ & $2^{5} .3^{8}$ & 54 \\
\hline 234 & $A_{2} A_{2} A_{1} A_{1} A_{1}$ & $2^{7} \cdot 3^{5}$ & 54 \\
\hline 235 & $A_{2} A_{2} A_{1} A_{1} A_{1}$ & $2^{7} \cdot 3^{4}$ & 54 \\
\hline 236 & complex conjugate of 235 & & \\
\hline 237 & $A_{2} A_{1} A_{1} A_{1} A_{1} A_{1} A_{1}$ & $2^{9} .3^{2}$ & 54 \\
\hline 238 & $A_{2} A_{1} A_{1} A_{1} A_{1} A_{1} A_{1}$ & $2^{9} \cdot 3^{3}$ & 54 \\
\hline 239 & complex conjugate of 238 & & \\
\hline 240 & $A_{2} A_{1} A_{1} A_{1} A_{1} A_{1}$ & $2^{8} \cdot 3^{3}$ & 48 \\
\hline 241 & $A_{1} A_{1} A_{1} A_{1} A_{1} A_{1} A_{1} A_{1}$ & $2^{14} .3$ & 48 \\
\hline 242 & $A_{1} A_{1} A_{1} A_{1} A_{1} A_{1} A_{1} A_{1}$ & $2^{10} .3^{2} .7$ & 48 \\
\hline 243 & complex conjugate of 242 & & \\
\hline 244 & $A_{2} A_{1} A_{1} A_{1} A_{1}$ & $2^{8} .3^{2}$ & 42 \\
\hline 245 & $A_{1} A_{1} A_{1} A_{1} A_{1} A_{1} A_{1}$ & $2^{9} .3^{2}$ & 42 \\
\hline 246 & $A_{1} A_{1} A_{1} A_{1} A_{1} A_{1} A_{1}$ & $2^{9} .3 .7$ & 42 \\
\hline 247 & complex conjugate of 246 & & \\
\hline 248 & $A_{2} A_{1} A_{1} A_{1}$ & $2^{6} .3^{5}$ & 36 \\
\hline 249 & $A_{1} A_{1} A_{1} A_{1} A_{1} A_{1}$ & $2^{9} .3^{2}$ & 36 \\
\hline 250 & $A_{2} A_{2}$ & $2^{6} \cdot 3^{7}$ & 36 \\
\hline 251 & $A_{3}$ & $2^{13} \cdot 3^{6}$ & 36 \\
\hline 252 & $A_{1} A_{1} A_{1} A_{1} A_{1}$ & $2^{7} .3 .5$ & 30 \\
\hline 253 & $A_{1} A_{1} A_{1} A_{1} A_{1}$ & $2^{8} .3^{2} .5$ & 30 \\
\hline 254 & complex conjugate of 253 & & \\
\hline 255 & $A_{1} A_{1} A_{1} A_{1}$ & $2^{9} .3^{2}$ & 24 \\
\hline 256 & $A_{1} A_{1} A_{1}$ & $2^{5} .3^{4}$ & 18 \\
\hline 257 & $A_{2}$ & $2^{4} .3^{6} .7 .13$ & 18 \\
\hline 258 & $A_{1} A_{1}$ & $2^{10} \cdot 3^{3}$ & 12 \\
\hline 259 & $A_{1}$ & $2^{4} .3^{2} .7 .13$ & 6 \\
\hline
\end{tabular}




\section{REFERENCES}

1. K. Abdukhalikov, Invariant Hermitian lattices in the Steinberg module and their isometry groups, Comm. Algebra 25 (8) (1997) 2607-2626. MR1459581(98e:20005)

2. K. Abdukhalikov, Unimodular Hermitian lattices in dimension 13, J. Algebra 272 (1) (2004) 186-190. MR2029031 (2005b:11098)

3. C. Bachoc, Applications of coding theory to the construction of modular lattices, J. Combin. Theory Ser. A 78 (1997) 92-119. MR1439633 (98a:11084)

4. W. Bosma, J. Cannon, C. Playoust, The Magma algebra system I: The user language, J. Symbolic Comput. 24 (3/4) (1997) 235-265. MR1484478

5. A. M. Cohen, Finite complex reflection groups, Ann. Sci. École Norm. Sup. (4) 9 (1976), no. 3, 379-436. MR0422448 (54:10437)

6. J. H. Conway, N. J. A. Sloane, Self-dual codes over $G F(3)$ and $G F(4)$ of length not exceeding 16, IEEE Trans. Inform. Theory 25 (1979) 312-322. MR528009 (80h:94026)

7. J. H. Conway, R. T. Curtis, S. P. Norton, R. A. Parker, R. A. Wilson, ATLAS of finite groups, Clarendon Press, Oxford, 1985. MR827219 (88g:20025)

8. J. H. Conway, N. J. A. Sloane, Sphere packings, lattices and groups, 3rd ed., Springer-Verlag New York, Inc., 1999. MR1662447 (2000b:11077)

9. R. Coulangeon, Minimal vectors in the second exterior power of a lattice, J. Algebra 194 (1997) 467-476. MR1467163 (98d:11078)

10. W. Feit, Some lattices over Q $(\sqrt{-3})$, J. Algebra 52 (1978) 248-263. MR0498407 (58:16534)

11. Y. Kitaoka, Arithmetic of quadratic forms, Cambridge Tracts in Math., 1993. MR1245266 (95c:11044)

12. M. Kneser, Klassenzahlen definiter quadratischer Formen, Arch. Math. 8 (1957) 241-250. MR0090606 (19:838c)

13. J. H. van Lint, F. J. MacWilliams, Generalized quadratic residue codes, IEEE Trans. Inform. Theory 24 (6) (1978) 730-737. MR.514352 (80a:94032)

14. F. G. MacWilliams, A. M. Odlyzko, N. J. A. Sloane, H. N. Ward, Self-dual codes over GF(4), J. Combin. Theory Ser. A 25 (1978) 288-318. MR514624 (80f:94016)

15. G. Nebe, Finite subgroups of $G L_{n}(\mathbb{Q})$ for $25 \leq n \leq 31$, Comm. Algebra 24 (7) (1996) 23412397. MR 1390378 (97e:20066)

16. W. Plesken, B. Souvignier, Computing isometries of lattices, Computational Algebra and Number Theory (London, 1993), J. Symbolic Comput. 24 (3/4) (1997) 327-334. MR1484483 (98i:11047)

17. H.-G. Quebbemann, Modular lattices in Euclidean spaces, J. Number Theory 54 (2) (1995) 190-202. MR1354045 (96i:11072)

18. U. Riese, On integral representations for SL(2, q), J. Algebra 242 (2001) 729-739. MR1848968 (2003c:20013)

19. R. Scharlau, R. Schulze-Pilot, Extremal lattices, in: B. H. Matzat, G.-M. Greuel, G. Hiss (Eds.), Algorithmic Algebra and Number Theory, Springer, 1998, 139-170. MR1672117 (99m:11074)

20. R. Scharlau, A. Schiemann, R. Schulze-Pilot, Theta series of modular, extremal, and hermitian lattices, Proceedings of the Conference on Integral and Quadratic Forms and Lattices, Seoul 1998, Contemporary Math. 249 (1999) 221-233. MR1732362(2001a:11066)

21. A. Schiemann, Classification of hermitian forms with the neighbour method, J. Symbolic Comput. 26 (4) (1998) 487-508. MR1646667(99g:11053)

22. G. C. Shephard, J. A. Todd, Finite unitary reflection groups, Canadian J. Math. 6 (1954) 274-304. MR0059914 (15:600b)

23. H. N. Ward, Quadratic residue codes and symplectic groups, J. Algebra 29 (1974) 150-171. MR $0444290(56: 2648)$

Institute of Mathematics, 125 Pushkin Str, 050010, Kazakhstan

E-mail address: abdukhalikov@math.kz

Department of Mathematics, University of Dortmund, 44221 Dortmund, Germany

E-mail address: Rudolf.Scharlau@math.uni-dortmund.de 